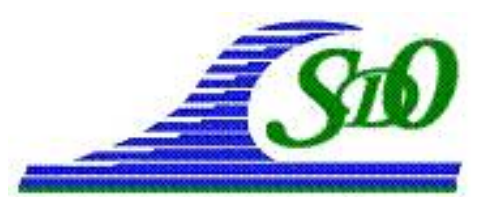

$X I^{\text {èmes }}$ Journées Nationales Génie Côtier - Génie Civil

Les Sables d'Olonne, 22-25 juin 2010

DOI:10.5150/jngcgc.2010.001-A @ Editions Paralia CFL

disponible en ligne - http://www.paralia.fr - available online

\title{
Observation et prévision des seiches sur la côte Atlantique française
}

\section{Fabrice ARDHUIN ${ }^{1}$, Emmanuel DEVAUX $^{2}$, Lucia PINEAU-GUILLOU ${ }^{3}$}

1. IFREMER, Centre de Brest, BP 70, 29280 Plouzané, France. ardhuin@ifremer.fr

2. CETE de l'Ouest, DIE/Groupe environnement, Man, rue René Viviani BP 46223, 44262 Nantes Cedex 2, France. emmanuel.devaux@developpement-durable.gouv.fr

3. SHOM, 13 rue du Chatellier, CS 92803, 29228 Brest Cedex 2, France lucia.pineau-guillou@shom.fr

\section{Résumé :}

Les seiches posent parfois problème pour la sécurité de la navigation, et peuvent entraver le bon fonctionnement des ports. Après un recensement des mesures analogiques ou numériques des marégraphes de ports français des côtes de l'Atlantique et de la Manche en 2007, le signal de seiche a été isolé. L’analyse fréquentielle a permis d'estimer les périodes propres à chaque port. L'analyse des causes possibles des seiches indique que, pour des seiches de périodes relativement courtes, comme à Port-Tudy avec 4 à 5 minutes, l'état de la mer est la cause dominante des seiches via l'excitation d'ondes infragravitaires à l'extérieur du port. Ainsi, la hauteur significative des ondes infragravitaires $\left(H_{\mathrm{IG}}\right)$ au sud de l'île de Groix est un indicateur fiable de la hauteur de la seiche (corrélation 0.87) et prévisible plusieurs jours en avance, grâce aux prévisions de spectres d'état de la mer.

\section{Mots-clés :}

Seiche, niveau de la mer - Etats de mer - Ondes infragravitaires - Marégraphie

\section{Introduction}

Les seiches ou modes de bassin, sont des ondes stationnaires dans un bassin fermé ou semi-fermé. Elles se manifestent par des oscillations de la hauteur d'eau, dont l'amplitude et la période varient selon la configuration du bassin. Leur amplitude est très variable dans le temps. Tous les lacs et ports ont des mouvements de seiche, dont les périodes sont caractéristiques de la forme des bassins (étendue et profondeur). Toutefois la seiche passe généralement inaperçue du fait de sa faible amplitude. Les grands ports ont des périodes de résonance de plusieurs dizaines de minutes, périodes auxquelles le forçage est généralement associé aux variations de pression atmosphérique (DE JONG et al., 2003). Les petits ports ont des périodes de résonance qui sont généralement inférieures à la dizaine de minutes, dans une bande de fréquence où les groupes de vagues génèrent un important signal infragravitaire. OKIHIRO et al. 
(1993) ont montré que la hauteur de la seiche pouvait atteindre 20 à 30 fois l'amplitude des ondes longues à l'entrée du port. Dans ces cas, l'agitation est maximale en présence de forts états de mer au large, avec une amplitude qui augmente avec la période de la houle: ainsi des seiches importantes peuvent survenir sans vent mais avec des houles de très grande période. D'autres situations océanographiques plus complexes peuvent aussi causer de fortes seiches (GIESE \& CHAPMAN, 2001).

Des seiches de hauteur notable (quelques dizaines de centimètres) se produisent à Brest, Le Conquet ou encore Cherbourg. Les seiches les plus importantes en France sont observées à Port-Tudy (Ile de Groix), où elles peuvent atteindre presque 2 mètres. Le CETMEF a réalisé en 2007 une enquête auprès des ports, avec l'appui du CETE Méditerranée, afin d'évaluer l'importance pratique du phénomène. Il en est ressorti que les problèmes les plus importants sont associés au déclenchement intempestif de la manœuvre automatique des portes d'écluse à Cherbourg, et aux problèmes de tenue à quai des navires du fait des vitesses associées à la seiche; c'est le cas à Bayonne et à Port-Tudy. Dans ce dernier port, la seiche, qui peut atteindre $1.8 \mathrm{~m}$ de hauteur, engendre des courants violents, pouvant dépasser 8 nœuds. Ainsi, le port est fermé à partir d'une hauteur de $1 \mathrm{~m}$, ce qui arrive plusieurs fois par an.

La gêne ou les dégâts occasionnés dans les ports français, ont donc motivé notre travail. Nous avons d'abord caractérisé les seiches puis cherché à déterminer leur cause. Ainsi, les mesures marégraphiques de l'année 2007 ont été analysées en relation avec les différents phénomènes susceptibles de générer les seiches. Ensuite, au vu de la forte corrélation entre les seiches observées et l'agitation infragravitaire générée par la houle, nous avons étudié la prévisibilité du phénomène, en particulier dans le cas de PortTudy.

\section{Analyse des signaux de seiche observés en 2007}

DEVAUX (2009) a analysé les données des marégraphes du SHOM de Brest, Cherbourg et Port-Tudy. Alors que les deux premiers sont numériques, ce qui facilite grandement le traitement, ce dernier est un marégraphe mécanique, enregistrant les hauteurs d'eau sur papier. C'est le dernier marégraphe mécanique du Réseau d'Observation du Niveau de la Mer du SHOM, conservé justement pour permettre d'enregistrer les seiches, quand les premiers marégraphes numériques avaient une période d'acquisition de 10 minutes. Il sera prochainement remplacé par un marégraphe numérique côtier.

Une méthode similaire est appliquée pour les enregistrements numériques : la hauteur de seiche est définie comme la différence entre le niveau maximal et le minimal sur une durée d'une heure, une fois enlevée la prédiction de marée. 


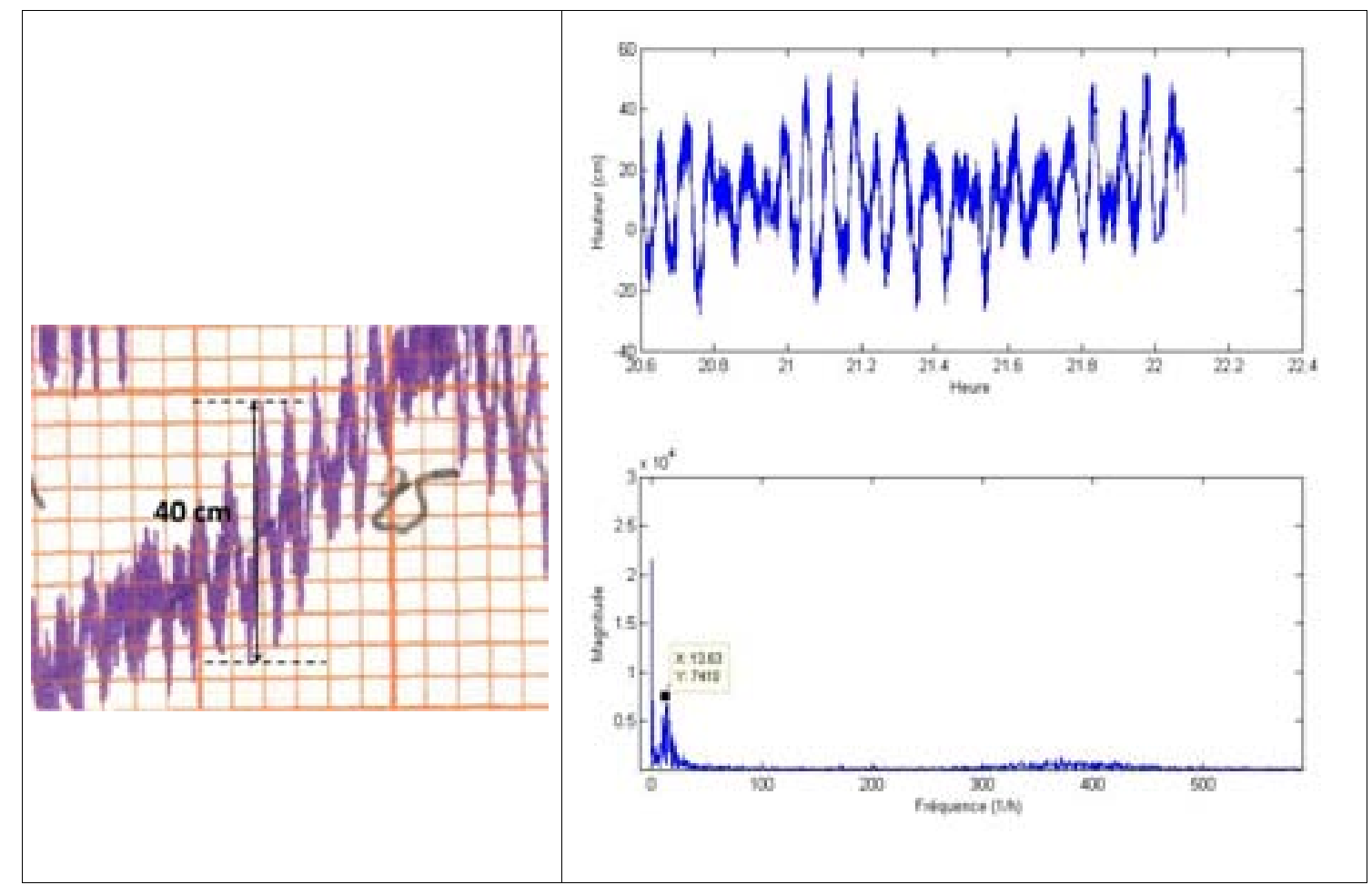

Figure 1. A gauche: illustration de l'estimation de la hauteur de seiche à Port-Tudy (cas du 25 février 2007). A droite: Signal (en haut) et spectre (en bas) de seiche (Port-Tudy, 16 janvier 2008).

A partir de ces signaux, nous avons étudié leur contenu fréquentiel par une analyse spectrale (figure 1). A Port-Tudy, la seiche présente un pic assez large autour de $13.6 \mathrm{~h}^{-1}=0,0038 \mathrm{~Hz}$ (période de 4.4 minutes), couvrant une bande de fréquence de $0.001 \mathrm{~Hz}$ environ. Les oscillations de fréquence $300 \mathrm{~h}^{-1}$ à $450 \mathrm{~h}^{-1}$ (périodes 8 à $12 \mathrm{~s}$ ) sont causées par la houle qui est tout de même bien filtrée par le port et le système de mesure. Une analyse similaire donne, dans le cas de Brest, des périodes de seiche de 7.5', 22', 85' et 230', pour Cherbourg 11', 20' et 37.5', pour Dieppe 17' et pour Le Conquet $4.5^{\prime}$ et $15^{\prime}$.

Les séries temporelles de hauteur de seiche sur l'ensemble des ports présentent de fortes similitudes, comme le montre la figure 2. Certains pics de seiche se retrouvent dans les quatre ports étudiés, avec des décalages de quelques heures seulement. Toutefois, la relation n'est pas systématique: en particulier le 21 février $(x=54)$, aucune seiche notable n'est observée à Cherbourg, contrairement aux autres ports. Il apparaît donc probable que les causes des seiches sont généralement homogènes spatialement, avec des exceptions pour certains événements. La corrélation entre deux séries temporelles ne dépasse pas 0.5 pour toute l'année 2007 (DEVAUX, 2009), et les corrélations les plus faibles sont obtenues entre Cherbourg et les autres ports. 


\section{Causes probables des seiches}

Au vu des études précédentes d’OKIHIRO et al. (1993) et de DE JONG et al. (2003), on peut penser que les états de mer, le vent ou la pression atmosphérique, sont la cause des seiches observées sur les côtes françaises. Le vent, la pression et les états de mer étant eux-mêmes corrélés, il n'est pas toujours aisé d'exclure l'un ou l'autre des facteurs. Toutefois, l'examen des pressions mesurées à Lann Bihoué et des vitesses du vent mesurées à Groix par Météo-France montre que la pression locale ou sa variation n'est pas significativement corrélée avec l'amplitude de la seiche à Port-Tudy. La vitesse du vent n'est pas un bon indicateur de la présence de seiches importantes, et la corrélation est typiquement inférieure à 0.5. Par contre, on peut noter que les fortes seiches à PortTudy $(\mathrm{H}>0.3 \mathrm{~m})$ se produisent généralement pour des vents de secteur sud-ouest, avec des azimuts compris entre 210 et $250^{\circ}$.

En absence de mesures d'état de mer au large de Port-Tudy, nous utiliserons ici les résultats des modèles de vagues développés au SHOM, tels que mis en œuvre dans le cadre de Prévimer, avec un dernier zoom utilisant un maillage à base triangulaire couvrant toute la Bretagne (figure 3). Ces modèles sont les plus précis à l'heure actuelle. La très bonne qualité des paramètres intégraux au large des côtes françaises (erreurs moyennes quadratiques inférieures à $9 \%$ et $8 \%$ des observations pour $H_{\mathrm{s}}$ et $T_{\mathrm{m} 0,2}$ moyennés sur trois heures à la bouée Brittany-62163), permet d'espérer une estimation correcte des ondes longues forcées. Nous utiliserons ici une série temporelle de spectres d'état de mer calculés pour un point au sud de l'île de Groix, par 28 m de fond (figure 3). L’état de la mer est généralement bien corrélé avec le vent local, sauf en présence de houle de longue période qui a pu être générée par une tempête très lointaine. L'analyse des seiches en été, saison où les houles longues dominent plus fréquemment l'état de la mer, montre sans ambiguïté que les seiches de Port-Tudy sont liées à la l'état de la mer. Ainsi la seiche du 1er juillet 2007, qui atteint $30 \mathrm{~cm}$ en milieu de journée, se produit au moment de l'arrivée d'une houle longue de période pic $16 \mathrm{~s}$ et de hauteur $1.8 \mathrm{~m}$, alors que le vent local est modéré (10 m/s), de secteur sud-ouest. Le 5 août 2007, une houle de période pic $18 \mathrm{~s}$ et de hauteur $0.4 \mathrm{~m}$ arrive en même temps qu'apparaît une seiche de $20 \mathrm{~cm}$. Dans ce, cas le vent est faible et orienté au sud-est.

En effet, l'état de la mer est souvent associé au vent local et les plus fortes vagues dans le secteur entre Lorient et Groix sont justement obtenues pour des directions des vagues (et donc généralement du vent) proche de $220^{\circ}$. En fait la corrélation est plus forte entre la direction des vagues et la hauteur de seiche qu'entre la direction du vent et la hauteur de seiche. 

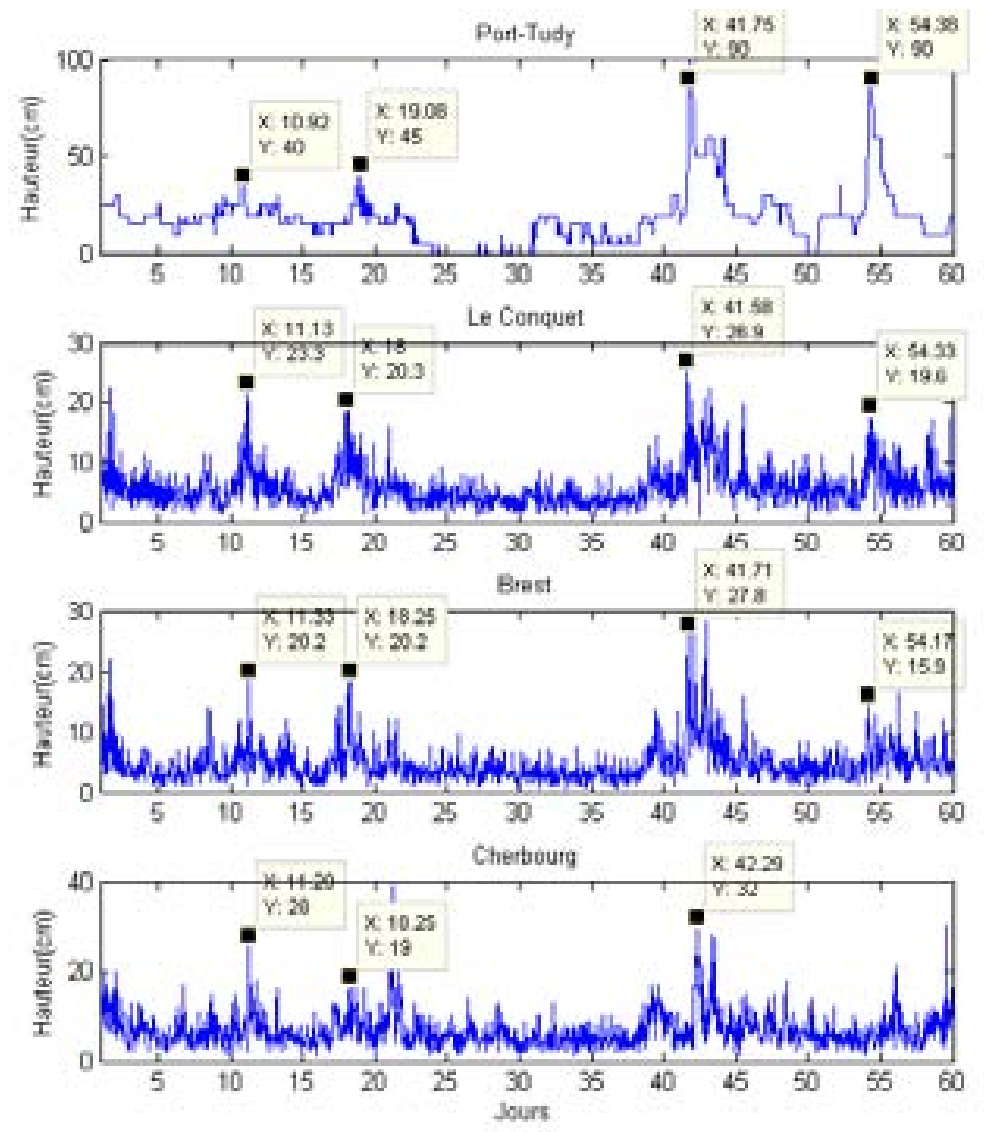

Figure 2. Comparaison des hauteurs de seiche entre Port-Tudy, Le Conquet, Brest et Cherbourg de janvier à février 2007.

\section{Prédiction quantitative de la hauteur des seiches à Port-Tudy}

Il est bien connu que les vagues génèrent des ondes longues (MUNK, 1949), aussi appelées "ondes infragravitaires". Ces ondes sont forcées par les groupes de vagues, avec une forte amplification à la côte. Au forçage local, s'ajoutent des ondes libres, généralement issues des ondes forcées mais libérées lors du déferlement à la côte (HERBERS, 1994). Des modèles relativement simples permettent de capturer les principaux traits de cette évolution (RENIERS et al., 2002). Ces ondes longues couvrent le domaine de fréquence de 0.001 à $0.05 \mathrm{~Hz}$, et sont donc susceptibles d'exciter les modes de bassin des ports, ce qui peut expliquer les seiches (OKIHIRO et al., 1993). Il est donc logique de chercher une relation entre la hauteur des seiches et l'amplitude des ondes longues de fréquences proches des seiches observées. Par ailleurs, l'amplitude du signal infragravitaire peut être reliée à des paramètres simples de l'état de la mer. Ainsi, pour un jeu de données regroupant un grand nombre de plages différentes, STOCKDON et al. (2006) ont obtenu une bonne corrélation entre l'amplitude des ondes infragravitaires à la côte et un indice de hauteur $H_{\mathrm{n}}=\operatorname{sqrt}\left(\mathrm{g}_{\mathrm{s}}\right) * \mathrm{~T}_{\mathrm{m} 0,-1}$, où $H_{\mathrm{s}}$ et $T_{\mathrm{m} 0,-1}$ 
sont, respectivement, la hauteur significative et la période moyenne (pondérée par le flux d'énergie) au large de la zone de déferlement.

Puisque les spectres d'état de mer $E(f, \theta)$ sont archivés toutes les trois heures, nous avons pris la moyenne quadratique des hauteurs de seiche sur un intervalle de trois heures. Une hauteur significative infragravitaire $H_{\mathrm{IG}}$, est obtenue en intégrant deux fois la variance de l'élévation de surface des ondes forcées dans la bande de fréquence 0.003-0.005 Hz, pour laquelle la seiche est observée à Port-Tudy. Le calcul est réalisé avec le coefficient de couplage $T\left(f_{1}, f_{2}, \theta\right)$, qui donne la densité spectrale de la variance de l'élévation de la surface, dans la bande infragravitaire :

$$
E_{2 d}(f)=2 \iiint T^{2}\left(f^{\prime}+f,-f, \theta-\theta^{\prime}\right) E\left(f^{\prime}+f, \theta\right) E\left(f^{\prime}, \theta^{\prime}\right) d f^{\prime} d \theta d \theta^{\prime}
$$

où $E(f, \theta)$ est la densité spectro-angulaire d'énergie des modes linéaires des vagues. Le coefficient de couplage $T$ est égal la moitié du coefficient $K$ donné par FORRISTALL (2000) et se déduit simplement des formules données par HASSELMANN (1962).

Du fait de la discrétisation relativement grossière de $E(f, \theta)$ dans le modèle, le calcul pratique de (1) demande de faire des hypothèses sur la variation du spectre à l'intérieur des intervalles de discrétisation. Nous avons pris $E(f, \theta)$ constant sur chaque élément spectral, et défini la hauteur significative infragravitaire forcée, comme :

$$
H_{I G}=4 \sqrt{\int_{f_{1}}^{f_{2}} E_{2 d}(f) d f}
$$

avec $\mathrm{f}_{1}=0.003$ et $\mathrm{f}_{2}=0.005 \mathrm{~Hz}$ qui encadrent la bande de fréquence de la seiche de PortTudy. La figure 3 montre la variation de la hauteur de seiche observée avec ce paramètre $H_{\mathrm{IG}}$ estimé pour le point de sortie du modèle d'état de mer. Le coefficient de corrélation linéaire de Pearson est de 0.87 entre les deux séries temporelles si on ne garde que les seiches de plus de $10 \mathrm{~cm}$. Pour l'année 2007, cela représente 1099 échantillons de 3 heures de durée, soit 38\% des cas.

Le paramètre $H_{\mathrm{IG}}$ peut donc être utilisé pour prévoir l'amplitude de la seiche. On remarque que toutes les seiches de plus de $0,5 \mathrm{~m}$ de hauteur -entraînant une gêne importante pour le fonctionnement du port - se sont produites pour $H_{\mathrm{IG}}>8 \mathrm{~cm}$ (24 cas en 2007). Si l'on devait utiliser une relation prédictive linéaire du type $H_{\text {seiche }}=3,8 H_{\mathrm{IG}}$ pour prévoir le dépassement du seuil $H_{\text {seiche }}=0,5 \mathrm{~m}$, cela aurait abouti à huit événements manqués sur 24, et 8 fausses alarmes. Toutefois, la hauteur de seiche minimale lors d'une fausse alarme aurait été de $30 \mathrm{~cm}$, soit une seiche assez importante pour perturber l'activité portuaire. Par ailleurs, on peut noter que la hauteur de seiche varie plutôt comme la racine carrée de $H_{\mathrm{IG}}$. Cela peut se comprendre assez simplement. Nous avons estimé $H_{\text {IG }}$ à une profondeur fixe de $20 \mathrm{~m}$, toujours située au large de la zone de déferlement. Or, plus la hauteur des vagues est faible, plus elles vont déferler à des profondeurs plus faibles, là ou les ondes longues forcées sont libérées et où la hauteur 


\section{XI $I^{\text {mes }}$ Journées Nationales Génie Côtier - Génie Civil \\ Les Sables d'Olonne, 22-25 juin 2010}

des vagues est plus importante. Cet effet pourrait expliquer une amplification relativement plus importante des petites valeurs de $H_{\mathrm{IG}}$.

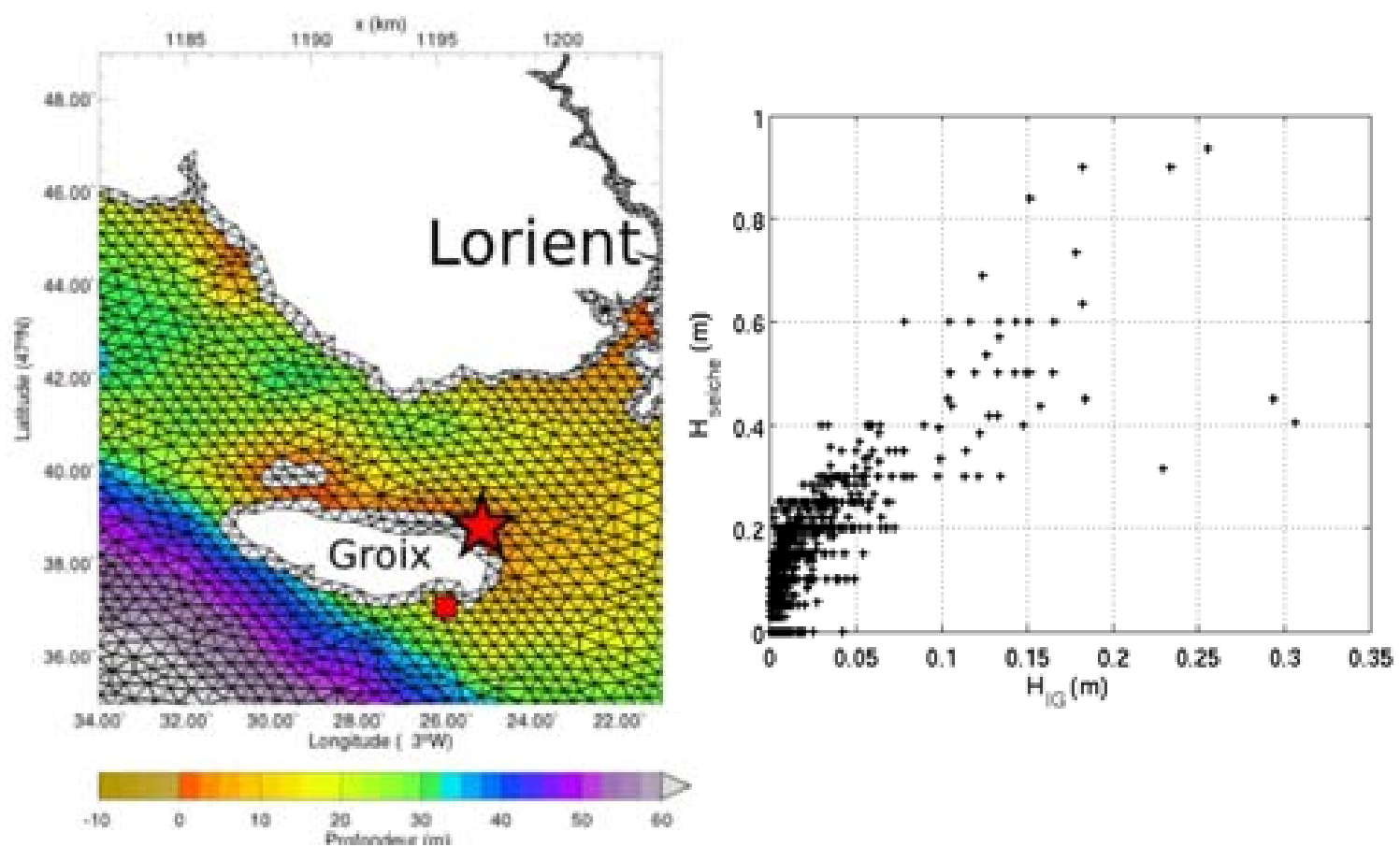

Figure 3. A gauche : maillage du modèle (Port Tudy est situé sur l'étoile rouge, le point de sortie pour le calcul de $H_{\mathrm{IG}}$ est sur le carré rouge). A droite: hauteurs de seiche observées en fonction de $H_{I G}$ modélisé (2007).

Il apparaît ainsi possible de prévoir la hauteur de seiche plusieurs jours à l'avance, sur la base de cette relation constatée entre l'état de la mer modélisé et la hauteur de seiche observée. Par ailleurs, il est intéressant de noter que l'indice de hauteur suggéré par STOCKDON et al. (2006), $H_{\mathrm{n}}=\operatorname{sqrt}\left(\mathrm{g}_{\mathrm{s}}\right) * \mathrm{~T}_{\mathrm{m} 0,-1}$ est bien corrélé avec la hauteur de seiche $(\mathrm{r}=0.77)$. Ce paramètre est facile à calculer à partir de paramètres qui sont bien prévus, jusqu'à 4 jours à l'avance, en particulier dans l'Atlantique nord-est.

\section{Conclusions}

L'analyse des hauteurs et périodes de seiche à Port-Tudy, Brest et Cherbourg a montré que le phénomène présente des caractéristiques propres à chaque port, en particulier en terme de périodes, mais que les seiches apparaissent souvent à peu près simultanément dans ces trois ports. L'analyse des causes probables de ces seiches montre que, dans le cas de Port-Tudy, l'état de la mer, via la génération d'ondes longues (infragravitaires) est probablement la cause unique de ces seiches dont les périodes sont proches de 4 minutes. Pour les seiches de périodes supérieures à 10 minutes à Brest, Le Conquet et Cherbourg, les corrélations sont plus faibles, entre 0.40 et 0.51 . Il est possible que 
d'autres causes se combinent à l'état de la mer, ou que la modélisation des états de mer ne soit pas de qualité suffisante pour prévoir cette partie du spectre des ondes longues.

\section{Références bibliographiques}

DE JONG M.P.C., HOLTHUIJSEN L.H., BATTJES J.A. (2003). Generation of seiches by cold fronts over the southern North Sea. Journal of Geophysical Research, vol. 108, 3117, 10 p. doi:10.1029/2002JC001422

DEVAUX E. (2009). Analyse des seiches à partir de l'exploitation de données marégraphiques, Rapport de projet de fin d'étude, ENTPE, Vaulx en Velin.

FORRISTALL G.Z. (2000). Wave crest distributions: observations and second-order theory. Journal of Physical Oceanography, vol. 30, pp 1931-1943. doi:10.1175/1520-0485(2000)030<1931:WCDOAS >2.0.CO;2

GIESE G.S., CHAPMAN D.C. (2001). Evidence of near-surface density stratification as a factor in extreme seiche events at Ciutadella harbor, Menorca Island. Proceedings of the $4^{\text {th }}$ International Symposium Ocean Wave Measurement and Analysis, pp 16151624, ASCE.

HASSELMANN H. (1962). On the non-linear energy transfer in a gravity wave spectrum, part 1: general theory. Journal of Fluid Mechanics, vol. 12, pp 481-501. doi:10.1017/S0022112062000373

HERBERS T.H.C. (1994). Infragravity-frequency (0.005-0.05 Hz) motions on the shelf, part I, forced waves. Journal of Physical Oceanography, vol. 24, pp 917-927. doi:10.1175/1520-0485(1994)024<0917:IFHMOT>2.0.CO;2

MUNK W. (1949). Surf beat. Eos Trans. AGU, vol. 30, pp 849-854.

OKIHIRO M., GUZA R.T., SEYMOUR R.J. (1993). Excitation of Seiche Observed in a Small Harbor. Journal of Geophysical Research, vol. 98, pp 18201-18211. doi:10.1029/93JC01760

RENIERS A.J.H.M., VAN DONGEREN A.R., BATTJES J.A., THORNTON E.B. (2002). Linear modeling of infragravity waves during DELILAH. Journal of Geophysical Research, vol. 107, 3137, 18 p. doi:10.1029/2001JC001083

STOCKDON H.F., HOLMAN R.A., HOWD P.A., SALLENGER A.H. (2006). Empirical parameterization of setup, swash, and runup. Coastal Engineering. vol. 53, pp 573-588. doi:10.1016/j.coastaleng.2005.12.005 